\title{
COMMENTARY
}

\section{Using lung ultrasound to differentiate patients in acute dyspnea in the prehospital emergency setting}

\author{
Joshua S Rempell* and Vicki E Noble \\ See related research by Prosen et al., http://ccforum.com/content/15/2/R114
}

\begin{abstract}
The diagnosis and treatment of dyspnea in the emergency department and in the prehospital setting is a challenge faced by the emergency physician and other prehospital care providers. While the use of lung ultrasound as a diagnostic tool in dyspneic patients has been well researched, there has been limited evaluation of its use in the prehospital setting. In the previous issue of Critical Care, Prosen and colleagues study the accuracy of lung ultrasound compared with both $\mathrm{N}$-terminal pro-brain natriuretic peptide and the clinical examination for differentiating between acute decompensated congestive heart failure and chronic obstructive pulmonary disease exacerbations for patients in the prehospital setting. Their article adds to the growing body of evidence demonstrating the diagnostic efficacy of lung ultrasound in differentiating between these two disease processes in the acutely dyspneic patient.
\end{abstract}

Acute dyspnea is a common, and often challenging, complaint both in the emergency department and in the prehospital setting. The physician or other care provider must often make treatment decisions based on limited information, without the time or resources to obtain diagnostic test results to help focus treatment. There has been growing interest and evidence supporting the use of lung ultrasound as a diagnostic tool to help differentiate the various causes of dyspnea, most specifically between patients with acute congestive heart failure and those with chronic obstructive pulmonary disease and asthma [1-5]. The current paper by Prosen and colleagues

*Correspondence: jrempell@partners.org

Department of Emergency Medicine, Massachusetts General Hospital, Boston, MA 02114, USA expands on previous work by evaluating the utility of ultrasound in the prehospital setting [1]. Their article is the first study to assess the utility of lung ultrasound compared with point of care $\mathrm{N}$-terminal pro-brain natriuretic peptide as well as specific clinical examination findings in the prehospital setting.

Unlike other forms of ultrasonography, lung ultrasound relies on the visualization of artifacts. Two artifacts, Alines and B-lines, are fundamental in the use of lung ultrasound. A-lines are bright horizontal hyperechoic lines deep to the pleural line that occur at regular intervals. These lines occur when the lung is well aerated and are a reverberation artifact. B-lines appear as vertical lines extending downward from the pleural line to the bottom of the ultrasound screen. B-lines appear when the interstitium becomes thickened with edema so that sound waves can now be reflected and refracted (instead of scattered) before returning to the probe.

Prosen and colleagues found that seeing B-lines on the initial lung ultrasound had 100\% sensitivity, 95\% specificity, $100 \%$ negative predictive value, and $96 \%$ positive predictive value for the diagnosis of heart failure in the prehospital setting. This was significantly superior to both $\mathrm{N}$-terminal pro-brain natriuretic peptide (92\% sensitivity and $89 \%$ specificity) as well as clinical examination ( $85 \%$ sensitivity and $86 \%$ specificity). One limitation of the current study is that the authors excluded patients who presented with noncongestive heart failure/ chronic obstructive pulmonary disease causes of dyspnea such as pneumonia, pulmonary embolus, pneumothorax, and pleural effusion. While pneumothorax and pleural effusion have been shown to be readily and accurately identified by lung ultrasound [6,7], it has also been shown that patients with focal infiltrates can have focal B-line patterns [8]. Including all patients with dyspnea will be important for further studies to truly characterize the utility of lung ultrasound in this setting.

More importantly, this study highlights the feasibility and ease of performing lung ultrasound. The lung ultrasound technique was feasible in $100 \%$ of patients in this 
study and all examinations took less than 1 minute to perform. This speed is one of the greatest strengths of lung ultrasound. Lung ultrasound is a diagnostic skill that can be easily performed, easily learned and used accurately $[8,9]$ - yet looking at outcomes will be important. There has been some evidence that the use of lung sonography has decreased the utilization of chest radiographs and computed tomography scans in the inpatient setting [10], so looking at outcomes and costeffectiveness in the outpatient setting would be very interesting. Moreover, it would also be interesting to look at the efficacy of diagnostic lung ultrasound in settings where currently no diagnostic imaging is available either because of geographical isolation or because of resource limitation. Given the relative steep learning curve for lung sonography and its reproducibility among users [9], the ability to make accurate diagnoses in the dyspneic patient when other diagnostic imaging is not available is a real medical advance.

In summary, the current study by Prosen and colleagues adds to the literature supporting the use of lung ultrasound in differentiating patients presenting with acute dyspnea. Lung ultrasound is an efficient, safe, feasible, and highly sensitive and specific test to aid in the diagnosis of the acutely dyspneic patient. This technique is a diagnostic modality extremely well suited to the prehospital setting, whether staffed by emergency physicians, paramedics, or other healthcare professionals. Further research, training, and application of lung ultrasound in the prehospital setting are warranted and are an exciting advancement for the field of emergency ultrasound.

\section{Competing interests}

The authors declare that they have no competing interests.
Published: 26 May 2011

\section{References}

1. Prosen G, Klemen P, Strnad M, Grmec S: Combination of lung ultrasound (a comet-tail sign) and $\mathrm{N}$-terminal pro-brain natriuretic peptide in differentiating acute heart failure from chronic obstructive pulmonary disease and asthma as cause of acute dyspnea in prehospital emergency setting. Crit Care 2011, 15:R114.

2. Lichtenstein D, Mézière G, Biderman P, Gepner A, Barré O: The comet-tail artifact. An ultrasound sign of alveolar-interstitial syndrome. Am J Respir Crit Care Med 1997, 156:1640-1646.

3. Licthenstein DA, Mezeire GA: Relevance of lung ultrasound in the diagnosis of acute respiratory failure: the BLUE protocol. Chest 2008, 134:117-125.

4. Volpicelli G, Mussa A, Garofalo G, Cardinale L, Casoli G, Perotto F, Fava C, Frascisco M: Bedside lung ultrasound in the assessment of alveolarinterstitial syndrome. Am J Emerg Med 2006, 24:689-696.

5. Liteplo AS, Marill KA, Villen T, Miller RM, Murray AF, Croft PE, Capp R, Noble VE: Emergency Thoracic Ultrasound in the Differentiation of the Etiology of Shortness of Breath (ETUDES): sonographic B-lines and N-terminal probrain-type natriuretic peptide in diagnosing congestive heart failure. Acad Emerg Med 2009, 16:201-210.

6. Rowan KR, Kirkpatrick AW, Liu D, Forkheim KE, Mayo JR, Nicolaou S: Traumatic pneumothorax detection with thoracic US: correlation with chest radiography and CT - initial experience. Radiology 2002, 225:210-214.

7. Grimberg A, Shigueoka DC, Atallah AN, Ajzen S, lared W: Diagnostic accuracy of sonography for pleural effusion: systematic review. Sao Paulo Med J 2010, 128:90-95.

8. Reissig A, Copetti R, Kroegel C: Current role of emergency ultrasound of the chest. Crit Care Med 2011. [Epub ahead of print].

9. Bedetti G, Gargani L, Corbisiero A, Frassi F, Poggianti E, Mottola G: Evaluation of ultrasound lung comets by hand-held echocardiography. CardiovasC Ultrasound 2006, 4:34.

10. Peris A, Tutino L, Zagli G, Batacchi S, Cianchi G, Spina R, Bonizzoli M, Migliaccio L, Perretta L, Bartolini M, Ban K, Balik M: The use of point-of-care bedside lung ultrasound significantly reduces the number of radiographs and computed tomography scans in critically ill patients. Anesth Analg 2010, 111:687-692.

\section{doi:10.1186/cc10226}

Cite this article as: Rempell JS, Noble VE: Using lung ultrasound to differentiate patients in acute dyspnea in the prehospital emergency setting. Critical Care 2011, 15:161. 